\title{
Observation of Pull-In Instability in Graphene Membranes under Interfacial Forces
}

\author{
Xinghui Liu, ${ }^{\dagger}$ Narasimha G. Boddeti, ${ }^{\dagger}$ Mariah R. Szpunar, ${ }^{\dagger}$ Luda Wang, ${ }^{\dagger}$ Miguel A. Rodriguez, ${ }^{\S}$ \\ Rong Long, ${ }^{\dagger}\|\|$ Jianliang Xiao, ${ }^{\dagger}$ Martin L. Dunn, ${ }^{\perp}$ and J. Scott Bunch ${ }^{*},{ }^{\dagger}$ \\ ${ }^{\dagger}$ Department of Mechanical Engineering, University of Colorado, Boulder, Colorado 80309, United States \\ ${ }^{\ddagger}$ Department of Mechanical Engineering, University of Miami, Coral Gables, Florida 33124, United States \\ ${ }^{\S}$ Department of Mechanical Engineering, Columbia University, New York, New York 10027, United States \\ "Department of Mechanical Engineering, University of Alberta, Edmonton, Alberta T6G 2G8, Canada \\ ${ }^{\perp}$ Singapore University of Technology and Design, Singapore, 138682
}

Supporting Information

ABSTRACT: We present a unique experimental configuration that allows us to determine the interfacial forces on nearly parallel plates made from the thinnest possible mechanical structures, single and few layer graphene membranes. Our approach consists of using a pressure difference across a graphene membrane to bring the membrane to within $\sim 10-20 \mathrm{~nm}$ above a circular post covered with $\mathrm{SiO}_{x}$ or $\mathrm{Au}$ until a critical point is reached whereby the membrane snaps into adhesive contact with the post. Continuous measurements of the deforming membrane with an AFM coupled with a theoretical model allow us to deduce the magnitude of the interfacial forces between graphene and $\mathrm{SiO}_{x}$ and graphene and $\mathrm{Au}$. The nature of the interfacial

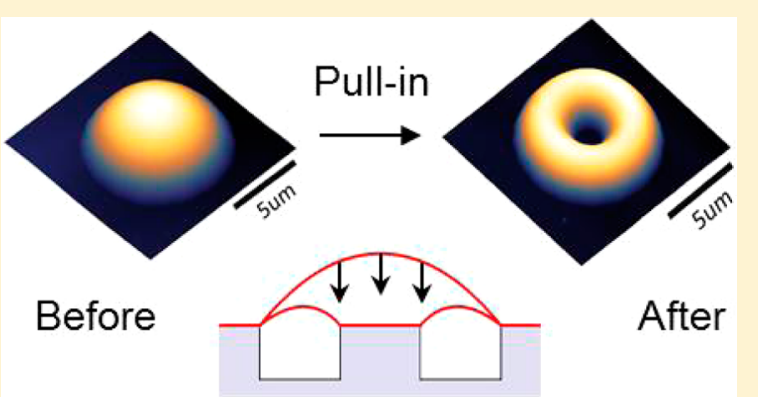
forces at $\sim 10-20 \mathrm{~nm}$ separation is consistent with an inverse fourth power distance dependence, implying that the interfacial forces are dominated by van der Waals interactions. Furthermore, the strength of the interactions is found to increase linearly with the number of graphene layers. The experimental approach can be used to measure the strength of the interfacial forces for other atomically thin two-dimensional materials and help guide the development of nanomechanical devices such as switches, resonators, and sensors.

KEYWORDS: Graphene, interfacial forces, nanoelectromechanical systems, pull-in instability

I nterfacial forces act between all materials. ${ }^{1}$ At macroscopic distances, these interfacial forces are weak and practically insignificant but at distances approaching tens of nanometers, they become much stronger, thereby enhancing the attraction within micro/nanomechanical structures or molecules, and potentially significantly affecting the device performance. ${ }^{2-5}$ Graphene, a two-dimensional nanomaterial composed of carbon atoms, is a promising material with potential applications in a variety of nanomechanical, biological, and electrical devices due to its exceptional properties..$^{6-14}$ Furthermore, graphene being extremely thin with a very high surface area to volume ratio is highly susceptible to interfacial forces and is an ideal candidate to study and characterize these forces. ${ }^{15,16}$ Therefore, there is an increasing interest in studying the nature of interfacial forces on graphene. ${ }^{17}$ Even though the adhesion strength between graphene and substrates when in contact has been experimentally measured in different ways, experimental measurements of noncontact attractive interfacial forces remains relatively unexplored. ${ }^{18-21}$ Interfacial forces on bulk materials or other nanomaterials have been measured using a variety of configurations. ${ }^{1,4,5,22}$ Here, we demonstrate a novel experimental method to study these elusive forces on graphene with a real time observation of the induced pull-in instability.

Devices used in this study consist of a graphene flake suspended over an annular ring etched into a silicon oxide wafer, forming a graphene-sealed microcavity (Figure 1a). Device configurations include graphene suspended on bare $\mathrm{SiO}_{x}$ or goldcoated $\mathrm{SiO}_{x}$. The graphene membranes are pressurized using a previously developed technique., ${ }^{7,}$ The suspended graphene membranes are placed in a high pressure chamber at a charging pressure, $p_{\text {ext }} \sim 300 \mathrm{kPa}$ of $\mathrm{H}_{2}$ gas, and left for a sufficiently long time $(\sim 10 \mathrm{~h})$ until the pressures inside, $p_{\text {int }}$ and outside of the microcavity, $p_{\text {ext }}$, equilibrate. After removing the sample from the high pressure chamber and bringing it to atmospheric pressure, a pressure difference, $\Delta p=p_{\text {int }}-p_{\text {ext }}$, exists across the graphene membrane. At low $\Delta p$, the graphene sheet remains adhered to the inner post and deforms in a donut shape (Figure 1b). At sufficiently high $\Delta p$, the force is large enough to overcome the adhesion energy of the graphene to the inner post, and the

Received: April 2, 2013

Revised: April 23, 2013

Published: April 24, 2013 


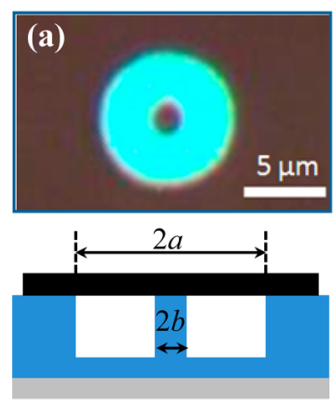

(d)

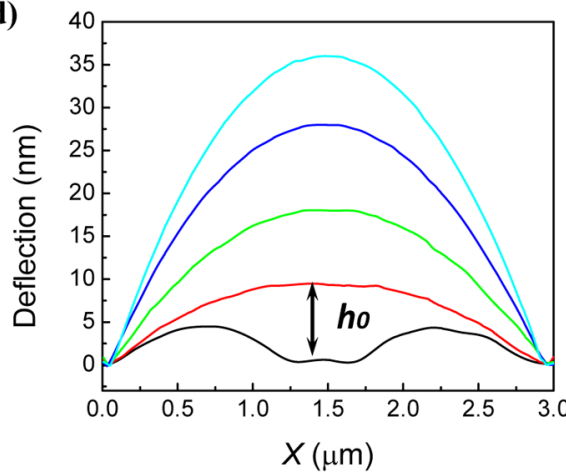

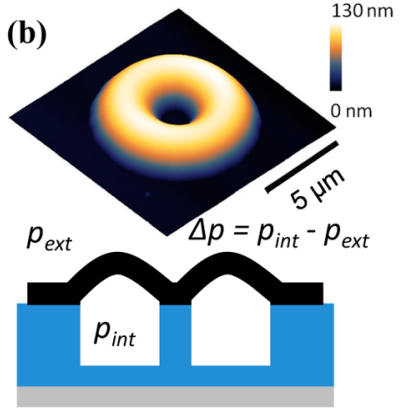

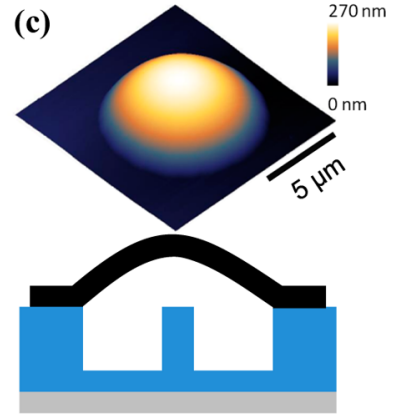

(e)

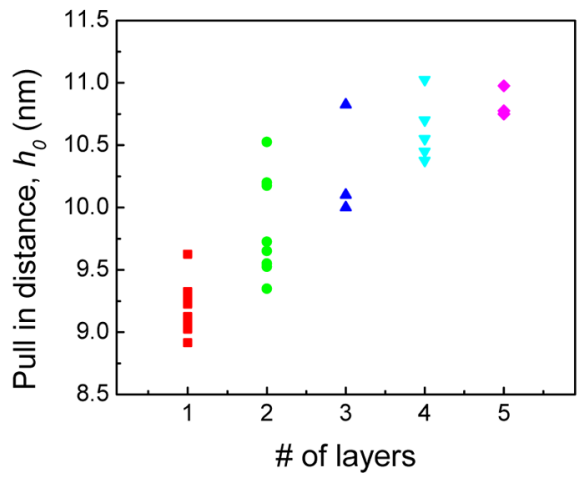

Figure 1. Measurement of the pull-in distance. (a) (upper) Optical image of suspended a few-layer graphene membrane in an annular ring geometry. (lower) Side view schematic of the suspended graphene on the annular ring. (b) (upper) A 3d rendering of an AFM image of a pressurized graphene membrane in the annular ring geometry before delamination from the inner post. (lower) Side view schematic of the pressurized suspended graphene on the annular ring. (c) (upper) A $3 \mathrm{~d}$ rendering of an AFM image of a pressurized graphene membrane in the annular ring geometry after delamination from the inner post. (lower) Side view schematic of the pressurized suspended graphene delaminated from the inner post. (d) A series of AFM line cuts through the center of a pressurized graphene membrane during pull-in. The outer diameter, $2 a=3 \mu \mathrm{m}$, and inner diameter, $2 b=0.5 \mu \mathrm{m}$. (e) Pull-in distance, $h_{0}$, vs number of layers for graphene membranes in an annular ring geometry with $2 a=3 \mu \mathrm{m}$ and $2 b=0.5 \mu \mathrm{m}$.
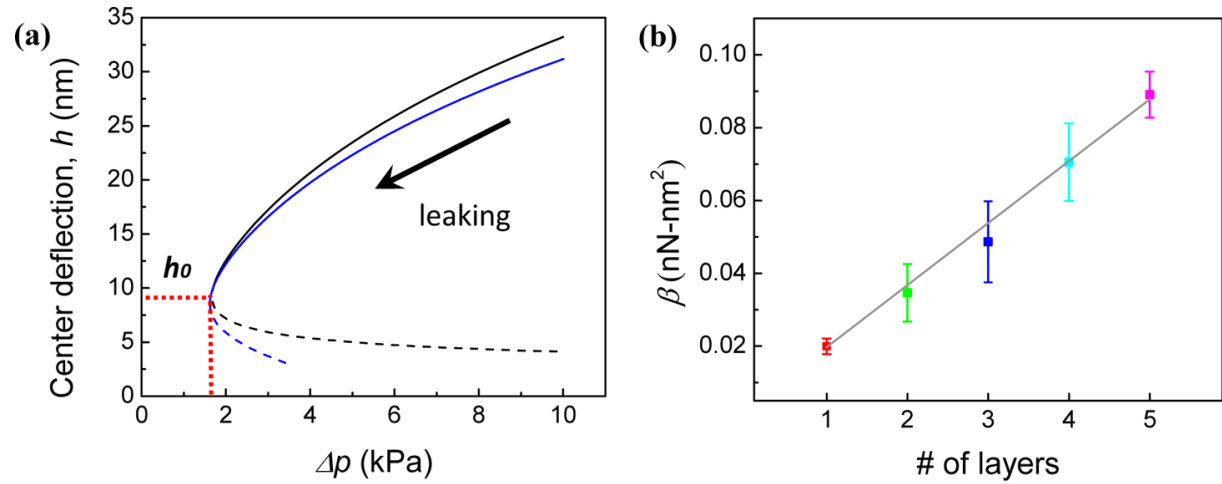

Figure 2. Scaling of $\beta$ with number of layers. (a) Center deflection, $h$, versus pressure difference, $\Delta p$, calculated for a monolayer graphene membrane in the annular ring geometry with an outer diameter, $2 a=3 \mu \mathrm{m}$, and inner diameter, $2 b=0.5 \mu \mathrm{m}$. The red dashed line at $\Delta p=1.68 \mathrm{kPa}$ corresponds to pullin and the deflection at this point is $h_{0}=9.2 \mathrm{~nm}$. The black line corresponds to the analytical model and the blue line is a finite element analysis model (b) The calculated values of $\beta$ vs number of layers using the data in (a) assuming a model where the force responsible for pull-in has the form $P_{\text {att }}=\beta / h^{4}$. The initial tension $S_{0}$ is assumed to be $0.07 \mathrm{~N} / \mathrm{m}$. A best fit line through the data is also shown which has a slope of $0.017 \mathrm{nN} \cdot \mathrm{nm}^{2} / \mathrm{number}$ of layer.

graphene membrane delaminates from it, becoming a spherical cap (Figure 1c).

After creating deformed spherical caps, our strategy is to then let gas slowly diffuse out of the microcavity through the underlying $\mathrm{SiO}_{x}$ substrate that decreases $\Delta p$ and the corresponding central deflection, $h$, of the graphene membrane until it is pulled back onto the center post due to attractive interactions between the post and graphene membrane. This process is monitored in real-time using an atomic force microscope, AFM (Figure $1 \mathrm{~d}$ and Supporting Information movie). Figure $1 \mathrm{~d}$ shows a series of AFM line scans through the center of a pressurized graphene membrane before and after the pull-in process. Initially a line trace through the center of the membrane (dark blue) corresponds to the situation in Figure 1c where the graphene is delaminated from the inner post. At a later time (black), the graphene is pulled onto the post and the graphene is deformed in a donut shape as seen in Figure $1 \mathrm{~b}$. The red line corresponds to a line trace just before pull-in. We call the center deflection at this point in time, the pull-in distance, $h_{0}$. Figure 1e shows the measured pull-in distance, $h_{0}$, versus number of graphene layers for graphene sheets in an identical geometry on the same chip (see Supporting Information). The number of graphene sheets was verified by Raman spectroscopy (see Supporting Information). The pull-in distance measured on bare 
(a)

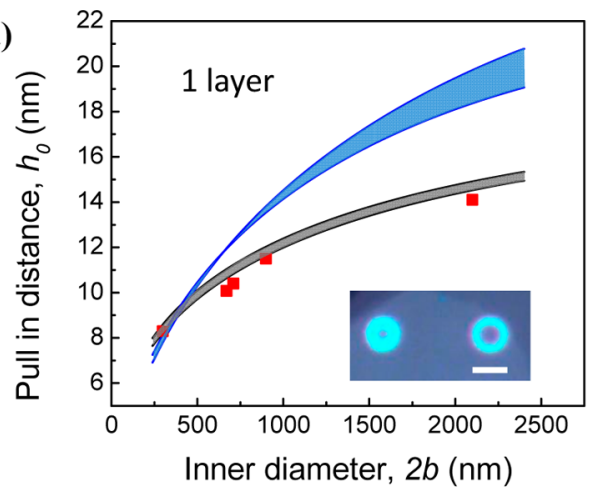

(c)

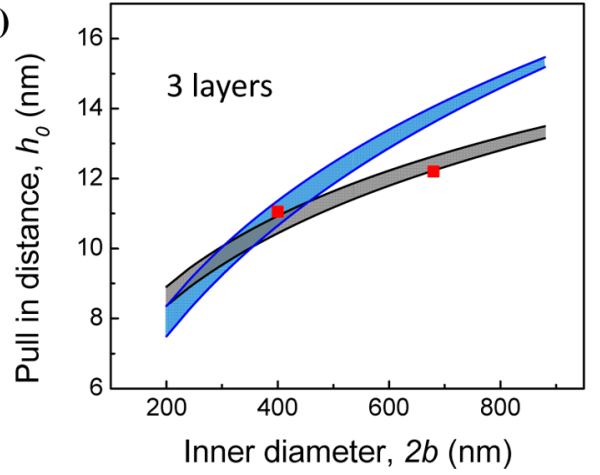

(b)

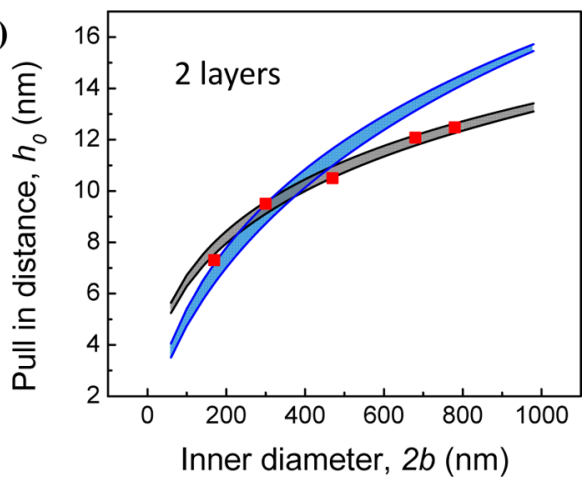

(d)

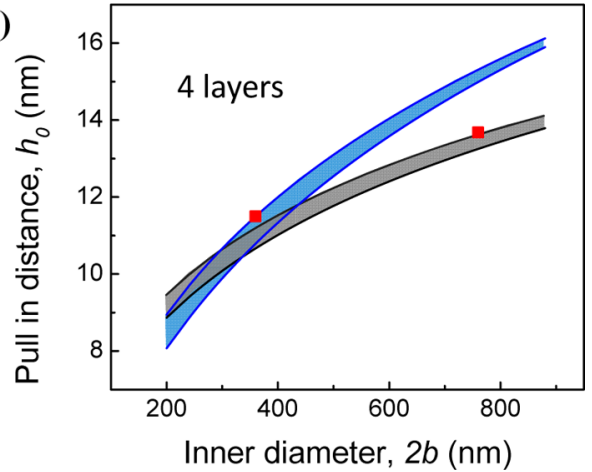

Figure 3. Scaling of the pull-in distance with $P_{\text {att. }}$ Pull-in distance, $h_{0}$, vs inner diameter, $2 b$, for (a) 1 layer (b) 2 layer (c) 3 layer (d) 4 layer graphene flakes (verified by Raman spectroscopy) with identical outer diameter but different inner diameters. The black and blue shaded lines are the calculated results for two different power law dependences $P_{\text {att }}=\beta / h^{4}$ (black) and $P_{\text {att }}=\alpha / h^{2}$ (blue) with $S_{0}=0.03-0.09 \mathrm{~N} / \mathrm{m}$. The values of $\beta$ and $\alpha$ are listed in Supporting Information. (a) (inset) Optical image of 2 of the measured monolayer devices. The scale bar $=5 \mu \mathrm{m}$.

$\mathrm{SiO}_{x}$ substrate, $h_{0}$, increases slightly with the number of layers from an average value of $h_{0}=9.2 \mathrm{~nm}$ for 1 layer graphene to $h_{0}=$ $10.8 \mathrm{~nm}$ for 5 layer graphene. At these values of $h_{0}$, the variation in the height of the graphene over the post is small and the post and graphene are effectively two parallel plates.

The pull-in behavior observed here is similar to the pull-in or jump-in of a cantilever spring into contact due to interfacial forces. ${ }^{4,23}$ We model the pull-in behavior in a continuum setup by considering an isotropic pressurized graphene membrane with initial surface tension, $S_{0}$, and an attractive pressure, $P_{\text {att }}$ due to the interfacial force between the post and the graphene membrane. ${ }^{18,24-27}$ The analysis culminates in a relationship between the system parameters given by

$$
\begin{aligned}
& \frac{E t}{32 a^{2}(1-v)}\left(\left(\Delta p-P_{\text {att }}\right)^{2} b^{4}+\Delta p^{2}\left(a^{4}-b^{4}\right)+P_{\text {att }}^{2} b^{4} \log \left(\frac{a^{4}}{b^{4}}\right)\right. \\
& \left.\left.-4 \Delta p P_{\text {att }} b^{2}\left(a^{2}-b^{2}\right)\right)+\left(S_{0}\left(\frac{1}{4 h}\left(\Delta p a^{2}-P_{\text {att }} b^{2}\left(1+\log \left(\frac{a^{2}}{b^{2}}\right)\right)\right)\right)^{2}\right)\right) \\
& =\left(\frac{1}{4 h}\left(\Delta p a^{2}-P_{\text {att }} b^{2}\left(1+\log \left(\frac{a^{2}}{b^{2}}\right)\right)\right)\right)^{3}
\end{aligned}
$$

where $E$ is the elastic modulus of graphene, $t$ is the thickness, $\nu$ is the Poisson ratio, and $a$ and $b$ are the outer and inner radii of the annular cavity, respectively. Equation 1 establishes a relationship between $h$ and $\Delta p$ if $S_{0}, E t, a, b$, and $P_{\text {att }}$ are known. The radii, $a$ and $b$, are measured by AFM, while $E t$ and $\nu$ are taken from wellestablished values in the literature for single and few layer graphene. ${ }^{7,18,27}$ We cannot directly measure $S_{0}$ so we assume values in the range of $S_{0}=0.03-0.15 \mathrm{~N} / \mathrm{m}$ with an average value of $S_{0}=0.07 \mathrm{~N} / \mathrm{m}$, consistent with numerous experimental measurements for exfoliated suspended graphene membranes in a similar geometry. ${ }^{7,28,29}$ Figure $2 \mathrm{a}$ shows the relationship between $h$ versus $\Delta p$ obtained from eq 1 using the system parameters for a monolayer graphene membrane: $a=1.5 \mu \mathrm{m}, b=$ $0.25 \mu \mathrm{m}, S_{0}=0.07 \mathrm{~N} / \mathrm{m}, E t=340 \mathrm{~N} / \mathrm{m}, \nu=0.16$, and $P_{\text {att }}=\beta / h^{4}$ $=0.0199 \mathrm{nN} \cdot \mathrm{nm}^{2} / h^{4}$. The deflection, $h$, decreases with decreasing $\Delta p$ (leaking gas) until a critical point is reached. At this critical maximum deflection, $h_{0}$, the graphene is sufficiently close to the post and pulled into the post by the attractive force. This pull-in instability is illustrated by the point on the curve where the slope goes to infinity at the pull-in distance $h_{0}$, or

$$
\left.\frac{\mathrm{d} \Delta p}{\mathrm{~d} h}\right|_{h=h_{0}}=0
$$

The measured $h_{0}, a$, and $b$, coupled with the values of $S_{0}, E t$, and $\nu$ taken from the literature, allow us to determine $P_{\text {att }}$ by solving eqs 1 and 2 simultaneously for $\Delta p$ and $P_{\text {att }}$. A comparison to a high-fidelity finite element model that more accurately treats the spatial dependence of the attractive forces is shown in blue on Figure 2a; the close agreement between them supports the validity of our analytical model.

We assumed an attractive force law of the form $P_{\text {att }}=\beta / h^{4}$, consistent with the van der Waals (vdW) force derived from Lifshitz theory between graphene and $\mathrm{SiO}_{2}$ for separations on the order of $10 \mathrm{~nm}$ or the phenomenological Lennard-Jones pair potential of interaction. $1,16,30-32$ From the experimentally measured pull-in distances in Figure le we calculate $\beta$ for each device and arrive at the corresponding $P_{\text {att }}\left(h=h_{0}\right)$. This is shown in Figure $2 \mathrm{~b}$ where $\beta=0.0199 \mathrm{nN} \cdot \mathrm{nm}^{2}$ for monolayer graphene. This value is $\sim 1.5 \%$ of the dispersion force between two perfectly metallic parallel plates, $P_{\text {att }}=\pi \hbar c / 240 h^{4}=1.3 \mathrm{nN} \cdot \mathrm{nm}^{2} / h^{4,33}$ and 
agrees reasonably well with recent theoretical calculations for graphene and $\mathrm{SiO}_{2}$ at $10 \mathrm{~nm}$ separations, $\beta=0.001-0.01$ $\mathrm{nN} \cdot \mathrm{nm}^{2}$ for an intrinsic graphene doping density of $10^{14} \mathrm{~m}^{-2}$ and $10^{16} \mathrm{~m}^{-2}$ at $T=300 \mathrm{~K}$, respectively. ${ }^{16}$ Figure $2 \mathrm{~b}$ also shows that $\beta$ increases linearly with the number of layers, up to five layers, with a slope of $0.017 \mathrm{nN} \cdot \mathrm{nm}^{2} /$ layer, close to the measured value of monolayer graphene, $\beta=0.0199 \mathrm{nN} \cdot \mathrm{nm}^{2}$. This increase with layer number suggests that the strength of the force is increasing in an integer manner as additional graphene layers are added. This is consistent with the additive nature of the vdW force. ${ }^{1,30}$ Our results are interesting in the context of recent experiments where an AFM tip was pulled off of a graphene substrate where the pull-off force was observed to depend on the number of graphene layers in suspended membranes ${ }^{34}$ but not on graphene supported by a substrate. ${ }^{34-36}$ Despite this similarity in response, we note that pull-off experiments are well-known to be different mechanistically than the pull-in experiments of our study.

In addition to vdW force, the interfacial forces can be from capillary or electrostatic forces. The capillary forces take effect when graphene membranes or the substrate are covered with liquid films and the liquid films touch, and the force can be described by $P_{\text {att }} \propto 1 / h^{1,30,31}$ However, we assume that the capillary force is not a likely candidate for the interfacial forces causing the pull-in phenomenon because absorbed liquid films of $10 \mathrm{~nm}$ thickness are unlikely to form between graphene membranes and the substrate. ${ }^{37,38}$ The electrostatic interaction, which can arise from image charges, work function differences or patch potentials can be described by $P_{\text {att }} \propto 1 / h^{2} \cdot{ }^{1,39,40}$ To further study the power law model considering different origins of the interaction, we varied the geometry of the annular ring. The pullin distance for 1-4 layers graphene membranes with an identical outer diameter but a different inner diameter is shown in Figure 3. The pull-in distance shows a slight increase with increasing $b$. A theoretical calculation based on our analytical model using $P_{\text {att }}=$ $\beta / h^{4}$ and the calculated values of $\beta$ in Figure $2 \mathrm{~b}$ is shown as a black shaded line in Figure 3. The boundaries of the shaded lines show the range of values for $S_{0}=0.03-0.09 \mathrm{~N} / \mathrm{m} .{ }^{29}$

To determine if electrostatic forces play a significant role in our measurements, we fit the data in Figures 1e and 3 with a model in which an electrostatic force takes the form, $P_{\text {att }}=\alpha / h^{2}$, and we use the same strategy to determine $\alpha$ as was used to calculate $\beta$ above. Doing so for the monolayer devices in Figure le gives $\alpha=$ $0.49 \mathrm{pN}$ (for $S_{0}=0.07 \mathrm{~N} / \mathrm{m}$ ). We can also use these values of $\alpha$ to fit the data in Figure 3. This is shown as a shaded blue line that fits poorly to the data. A good fit would require that $\alpha$ increases with inner post diameter for all the devices measured, while no such assumption is needed for $\beta$. To fit all of our measured pull-in distances (51 devices in 17 geometries from 5 different chips) using an electrostratic force model requires that $\alpha$ values vary from $0.15-1.79 \mathrm{pN}$ across all the devices. A model based on an inverse third power dependence was also examined and does not fit all the data as well as the inverse fourth power dependence (see Supporting Information).

To test the material dependence of the interfacial interaction with graphene, we also carried out experiments where we measured the pull-in distance between graphene and a gold coated annular ring that were electrically contacted and grounded (see Supporting Information). The 2-5 layers graphene membranes ( 17 devices in 6 similar geometries from 4 chips) were measured. The pull-in distance varied between 9 and $18 \mathrm{~nm}$ for annular rings with $a=1-1.75 \mu \mathrm{m}$ and $b=0.15-$ $0.6 \mu \mathrm{m}$, slightly larger than the measured pull-in distances for uncoated $\mathrm{SiO}_{x}$ posts of a similar geometry. Using the same theoretical analysis as with the graphene $/ \mathrm{SiO}_{x}$ data, we determined the average value and standard deviation of $\beta$ / number of graphene layers between the Au-coated post and electrically grounded graphene to be $=0.104 \pm 0.031 \mathrm{nN} \cdot \mathrm{nm}^{2} /$ layer; these are about an order of magnitude higher than those for graphene interacting with $\mathrm{SiO}_{x}$ (Figure 4). The graphene/Au

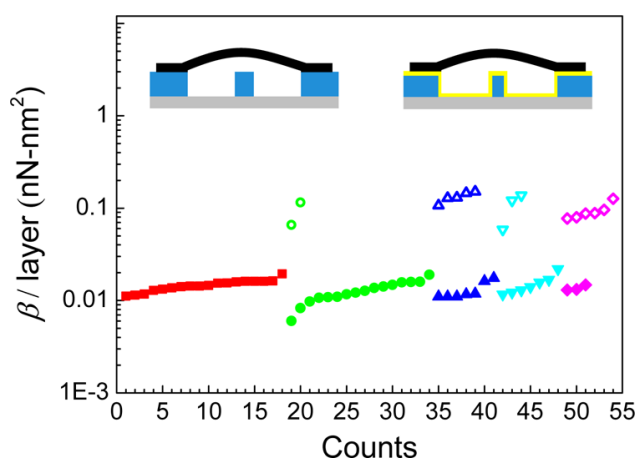

Figure 4. Modeled vdW force versus number of layers for $\mathrm{SiO}_{x}$ and gold. Measured $\beta$ /number of graphene layers between $\mathrm{SiO}_{x}$ and 1 layer graphene (solid red squares), 2 layer graphene (solid green circles), 3 layer graphene (solid blue up triangles), 4 layer graphene (solid cyan down triangles), 5 layer graphene (solid magenta diamond), and $\beta /$ number of graphene layers between $\mathrm{Au}$ and 2 layer graphene (hollow green circles), 3 layer graphene (hollow blue up triangles), 4 layer graphene (hollow cyan down triangles), and 5 layer graphene (hollow magenta diamond). The average and standard deviation of $\beta /$ number of graphene layers between $\mathrm{SiO}_{x}$ and graphene are $0.0179 \pm 0.0037$ $\mathrm{nN} \cdot \mathrm{nm}^{2} /$ layer. The average and standard deviation of $\beta /$ number of graphene layers between $\mathrm{Au}$ and graphene are $0.104 \pm 0.031 \mathrm{nN} \cdot \mathrm{nm}^{2}$ / layer. Each data point corresponds to a separate device. (top left inset) Side view schematic of the pressurized suspended graphene on the annular ring with $\mathrm{SiO}_{x}$ surface. (top right inset) Side view schematic of the pressurized suspended graphene on an Au coated annular ring.

values agrees reasonably well with the theoretical predictions based on a Lifshitz formula of graphene interacting with gold at $15 \mathrm{~nm}$ separation, $\beta=0.08 \mathrm{nN}-\mathrm{nm}^{2}$.

In conclusion, we observed the pull-in instability at $10-20 \mathrm{~nm}$ distance on graphene by the attractive interfacial forces between graphene and $\mathrm{SiO}_{x} / \mathrm{Au}$ and found them to agree very well with a form $P_{\text {att }}=\beta / h^{4}$, consistent with recently calculated values of long-range vdW forces between graphene and $\mathrm{SiO}_{x}$ and graphene and gold. Furthermore, the strength of the force scales linearly with layer numbers, which is compatible with the additive nature of $\mathrm{vdW}$ forces. It is noteworthy that our experimental configuration is essentially a realization of a parallel plate geometry by self-alignment to measure interfacial forces acting on atomically thin, two-dimensional materials. ${ }^{41}$ These experiments that provide a measurement of the magnitude and power law dependence of the interfacial forces at $10-20 \mathrm{~nm}$ separations between graphene and 2 common substrates can guide the development of nanomechanical devices from single and few layer graphene sheets where these forces are critical to their effective operation. ${ }^{6,42,43}$

\section{ASSOCIATED CONTENT}

\section{S Supporting Information}

Fabrication processes, counting the number of graphene layers, analytical model, finite element simulations, calculation of $\beta$, calculation of $\alpha, \gamma$, and deformation of graphene membrane by $\mathrm{vdW}$ force. This material is available free of charge via the Internet at http://pubs.acs.org. 


\section{AUTHOR INFORMATION}

\section{Corresponding Author}

*E-mail: jbunch@colorado.edu.

Notes

The authors declare no competing financial interest.

\section{ACKNOWLEDGMENTS}

We thank Rishi Raj for use of the Raman microscope. This work was supported by NSF Grants \#0900832(CMMI: Graphene Nanomechanics: The Role of van der Waals Forces), \#1054406(CMMI: CAREER: Atomic Scale Defect Engineering in Graphene Membranes), the DARPA Center on Nanoscale Science and Technology for Integrated Micro/Nano-Electromechanical Transducers (iMINT), the National Science Foundation (NSF) Industry/University Cooperative Research Center for Membrane Science, Engineering and Technology (MAST), and in part by the NNIN and the National Science Foundation under Grant ECS-0335765.

\section{REFERENCES}

(1) Israelachvili, J. N. Intermolecular And Surface Forces. Academic Press: 2010

(2) DelRio, F. W.; de Boer, M. P.; Knapp, J. A.; David Reedy, E.; Clews, P. J.; Dunn, M. L. Nat. Mater. 2005, 4 (8), 629-634.

(3) Maboudian, R; Howe, R. T. J. Vac. Sci. Technol., B 1997, 15 (1), $1-$ 20.

(4) Israelachvili, J. N.; Tabor, D. Proc. R. Soc. London, Ser. A 1972, 331 (1584), 19-38.

(5) Schneeweiss, P.; Gierling, M.; Visanescu, G.; Kern, D. P.; Judd, T. E.; Gunther, A.; Fortagh, J. Nat. Nanotechnol 2012, 7 (8), 515-519.

(6) Bunch, J. S.; van der Zande, A. M.; Verbridge, S. S.; Frank, I. W.; Tanenbaum, D. M.; Parpia, J. M.; Craighead, H. G.; McEuen, P. L. Science 2007, 315 (5811), 490-493.

(7) Bunch, J. S.; Verbridge, S. S.; Alden, J. S.; van der Zande, A. M.; Parpia, J. M.; Craighead, H. G.; McEuen, P. L. Nano Lett. 2008, 8 (8), $2458-2462$.

(8) Geim, A. K. Science 2009, 324 (5934), 1530-1534.

(9) Geim, A. K.; Novoselov, K. S. Nat. Mater. 2007, 6 (3), 183-191.

(10) Garaj, S.; Hubbard, W.; Reina, A.; Kong, J.; Branton, D.; Golovchenko, J. A. Nature 2010, 467 (7312), 190-193.

(11) Schneider, G. g. F.; Kowalczyk, S. W.; Calado, V. E.; Pandraud, G. g.; Zandbergen, H. W.; Vandersypen, L. M. K.; Dekker, C. Nano Lett. 2010, 10 (8), 3163-3167.

(12) Novoselov, K. S.; Geim, A. K.; Morozov, S. V.; Jiang, D.; Zhang, Y.; Dubonos, S. V.; Grigorieva, I. V.; Firsov, A. A. Science 2004, 306 (5696), 666-669.

(13) Chen, C.; Rosenblatt, S.; Bolotin, K. I.; Kalb, W.; Kim, P.; Kymissis, I.; Stormer, H. L.; Heinz, T. F.; Hone, J. Nat. Nanotechnol. 2009, 4 (12), 861-867.

(14) Xu, Y.; Chen, C.; Deshpande, V. V.; DiRenno, F. A.; Gondarenko, A.; Heinz, D. B.; Liu, S.; Kim, P.; Hone, J. Appl. Phys. Lett. 2010, 97 (24), 243111-3.

(15) Bordag, M.; Geyer, B.; Klimchitskaya, G. L.; Mostepanenko, V. M. Phys. Rev. B 2006, 74 (20), 205431.

(16) Sarabadani, J.; Naji, A.; Asgari, R.; Podgornik, R. Phys. Rev. B 2011, 84 (15), 155407.

(17) Bunch, J. S.; Dunn, M. L. Solid State Commun. 2012, 152 (15), 1359-1364.

(18) Koenig, S. P.; Boddeti, N. G.; Dunn, M. L.; Bunch, J. S. Nat. Nanotechnol. 2011, 6 (9), 543-546.

(19) Zong, Z.; Chen, C.-L.; Dokmeci, M. R.; Wan, K.-t. J. Appl. Phys. 2010, 107 (2), 026104-3.

(20) Yoon, T.; Shin, W. C.; Kim, T. Y.; Mun, J. H.; Kim, T.-S.; Cho, B. J. Nano Lett. 2012, 12 (3), 1448-1452.

(21) Scharfenberg, S.; Mansukhani, N.; Chialvo, C.; Weaver, R. L.; Mason, N. Appl. Phys. Lett. 2012, 100 (2), 021910-3.
(22) Lamoreaux, S. K. Phys. Rev. Lett. 1997, 78 (1), 5-8.

(23) Tabor, D.; Winterton, R. H. S. Proc. R. Soc. London, Ser. A 1969, 312 (1511), 435-450.

(24) Atalaya, J.; Isacsson, A.; Kinaret, J. M. Nano Lett. 2008, 8 (12), 4196-4200.

(25) Bao, W.; Miao, F.; Chen, Z.; Zhang, H.; Jang, W.; Dames, C.; Lau, C. N. Nat. Nanotechnol. 2009, 4 (9), 562-566.

(26) Duan, W. H.; Wang, C. M. Nanotechnology 2009, 20 (7), 075702.

(27) Lee, C.; Wei, X.; Kysar, J. W.; Hone, J. Science 2008, 321 (5887), $385-388$.

(28) Barton, R. A.; Ilic, B.; van der Zande, A. M.; Whitney, W. S.; McEuen, P. L.; Parpia, J. M.; Craighead, H. G. Nano Lett. 2011, 11 (3), $1232-1236$

(29) Wang, L.; Travis, J. J.; Cavanagh, A. S.; Liu, X.; Koenig, S. P.; Huang, P. Y.; George, S. M.; Bunch, J. S. Nano Lett. 2012, 12 (7), 37063710.

(30) Hans-Jurgen Butt, M. K. Surface and Interfacial Forces; WileyVCH: Germany, 2010.

(31) Bhushan, B. Handbook of Micro/Nano Tribology, 2nd ed.; CRC Press: Boca Raton, FL, 1998.

(32) Aitken, Z. H.; Huang, R. J. Appl. Phys. 2010, 107 (12), 12353110

(33) Casimir, H. On the attraction between two perfectly conducting plates. Proc. K. Ned. Akad. Wet 1948, 51 (793), 150.

(34) Deng, Z.; Klimov, N. N.; Solares, S. D.; Li, T.; Xu, H.; Cannara, R. J. Langmuir 2012, 29 (1), 235-243.

(35) Li, Q.; Lee, C.; Carpick, R. W.; Hone, J. Phys. Status Solidi B 2010, 247 (11-12), 2909-2914.

(36) Liu, X. Z.; Li, Q.; Egberts, P.; Zhang, B.,; Carpick, R. W. In Adhesion between nanoscale asperities and graphene: A study of twodimensional atomic membranes using atomic force microscopy (AFM). Proceedings of the 36th Annual Meeting of the Adhesion Society, Dayton Beach, FL, March 3-6, 2013.

(37) Beaglehole, D.; Christenson, H. K. J. Phys. Chem. 1992, 96 (8), 3395-3403.

(38) Zitzler, L.; Herminghaus, S.; Mugele, F. Phys. Rev. B: Condens. Matter 2002, 66 (15), 155436.

(39) Speake, C. C.; Trenkel, C. Phys. Rev. Lett. 2003, 90 (16), 160403.

(40) Kim, W. J.; Sushkov, A. O.; Dalvit, D. A. R.; Lamoreaux, S. K. Phys. Rev. A 2010, 81 (2), 022505.

(41) Bressi, G.; Carugno, G.; Onofrio, R.; Ruoso, G. Phys. Rev. Lett. 2002, 88 (4), 041804.

(42) Milaninia, K. M.; Baldo, M. A.; Reina, A.; Kong, J. Appl. Phys. Lett. 2009, 95 (18), 183105-3.

(43) Loh, O. Y.; Espinosa, H. D. Nat. Nanotechnol. 2012, 7 (5), $283-$ 295. 